Acta Hispanica (2020) Supplementum II: 835-845

\title{
LA EXTENSIÓN DE LA JORNADA ESCOLAR EN MÉXICO Y SUS EFECTOS EN LOS MAESTROS DE PRIMARIA
}

\author{
MANUEl SÁNCHEZ CERÓN \\ Universidad Pedagógica Nacional, Ciudad de México \\ Adriana García MEZA \\ Escuela Normal Superior del Estado de Puebla \\ Aidé Ávila Ayala \\ Universidad Pedagógica Nacional, Ciudad de México
}

\begin{abstract}
Resumen: En las últimas décadas las reformas educativas neoliberales han avanzado significativamente en América Latina. Los aspectos que han tocado son variados y van desde los que modifican el currículo hasta los que afectan las condiciones de trabajo de los maestros. Uno de estos cambios es la instauración de las escuelas de tiempo completo (ETC). En esta perspectiva, este artículo presenta un estudio exploratorio sobre los efectos que esta política ha tenido para los maestros de primaria en una entidad en México. El trabajo se basó en entrevistas focalizadas a cuatro profesores que trabajan en ETC. Sin embargo, como el fenómeno es muy complejo este trabajo solo considera tres niveles: a) los aspectos pedagógicos; b) el desgaste y el cansancio de los maestros; y, c) los efectos en los salarios.
\end{abstract}

Palabras clave: políticas educativas, escuelas de tiempo completo, educación básica, docentes, trabajo docente.

Abstract: In recent decades, neoliberal educational reforms have advanced significantly in Latin America. The aspects that have touched on these transformations are varied for example: those that modify the curriculum to those that affect the working conditions of teacher. One of these changes is the implementation of Full-time schools. In this perspective, this paper presents an exploratory study that shows the effects that this policy has had for primary school teacher in a entity in Mexico. The work was based on focused interviews with four teachers working in Fulltime schools. However, since the phenomenon is very complex, in this paper, we consider only three levels of analysis: a) the pedagogical aspects; b) wear and fatigues of teachers; and, c) the effects on wages.

Keywords: Education Policies, Full-Time Schools, Elementary School, Teachers, Teaching Work. 
La extensión de la jornada escolar en México y sus efectos en los maestros de primaria

\section{Introducción}

La política de ampliación de la jornada escolar en las instituciones de educación básica en América Latina no es una propuesta reciente. Más bien se ha ido poniendo en marcha sistemáticamente en las tres últimas décadas. Este fenómeno involucra uno de los más importantes elementos de la estructura tradicional de la distribución del tiempo en la escuela: las Escuelas de Tiempo Completo (ETC).

Los componentes estructurales de la vida escolar se están constituyendo en objeto de análisis y de investigación; porque se busca explicar cómo los cambios en la estructura escolar, en un contexto de profundas transformaciones del modelo económico de desarrollo está afectando a los maestros latinoamericanos.

Algunos investigadores como Baquero et al (1996), Bosco (2000) y Dussel (2006), entre otros, han identificado algunos elementos estructurales invariables de la vida en las escuelas; sin embargo, estos estudios se dirigen hacia dos ámbitos importantes de la práctica escolar: uno, los análisis orientados por la categoría de gramática escolar entendida como la configuración de aquellos elementos que integran la vida cotidiana de los niños y maestros en las escuelas (Tyack y Cuban: 1995); y, dos, la concepción foucaultiana de dispositivo ${ }^{1}$ que también puede utilizarse para el análisis de la estructura escolar.

En esta perspectiva, el concepto de gramática escolar posibilita la explicación del comportamiento de la escuela ante las reformas; en particular las impuestas en las últimas tres décadas en América Latina. Por ejemplo, los estudios de Hargreaves et al. (2008) y Hargreaves (2009) y, para el caso latinoamericano el análisis de Romero (2006), entre otros, son importantes para entender el fenómeno.

Este estudio exploratorio tuvo como objetivo identificar algunos efectos que el programa de ETC ha tenido para los maestros de primaria en Tlaxcala, México. Para el trabajo se seleccionaron a dos maestras y dos maestros que se desempeñan en ETC en el municipio de Ixtenco en Tlaxcala. Este municipio se encuentra ubicado al oriente del estado. La zona escolar en la que trabajan estos maestros está compuesta por ocho escuelas de las cuales cinco están incorporadas a este programa.

Metodológicamente, el trabajo se basó en entrevistas focalizadas y las temáticas giraron en torno a la experiencia que viven estos docentes en estas escuelas El análisis también se apoyó en una revisión de los documentos oficiales de este proyecto. El estudio se presenta en dos apartados; el primero, revisa la experiencia latinoamericana de las ETC y la propuesta en México; y el segundo, identifica tres rasgos de la experiencia de los maestros: los niveles pedagógicos, el desgaste y el cansancio de los maestros y los aspectos salariales.

\footnotetext{
${ }^{1}$ Foucault nunca dedicó un trabajo particular sobre la educación, pero el concepto de dispositivo se ha venido utilizando en relación, por ejemplo, con la aplicación de exámenes estandarizados y de carácter nacional empleados como mecanismos de control. Agamben (2011), por su parte, señala que el dispositivo es uno de los términos más importantes en el pensamiento teórico de Foucault; porque esta noción está asociada a lo que él llamó gobernabilidad o; en otros términos, el gobierno de los hombres.
} 
Manuel Sánchez Cerón - Adriana García Meza - Aidé Ávila Ayala

\section{La jornada escolar ampliada: algunos antecedentes en América Latina}

El debate actual sobre la puesta en marcha de las ETC en América Latina se puede agrupar, según los objetivos declarados, en cuatro principales orientaciones (Ramírez, 2014: 15):

a) La tendencia a asociar el aumento de horas de clase con el mejoramiento de resultados derivados de exámenes estandarizados nacionales (Martinic - Villalta, 2013; CIPPEC, 2006).

b) Su propósito es la contención social asociada a la vulnerabilidad que enfrentan los niños y niñas en América Latina.

c) Aquellos que buscan la institucionalización del control social de los menores a través de las escuelas.

d) Acercarse a las estadísticas de otros sistemas educativos que han aumentado sus puntajes en sus resultados.

Sin embargo, aunque la política de extensión de las jornadas de trabajo de los docentes de educación básica en la región no es reciente conviene destacar que en algunos casos ha sido, en cierto sentido, aislada. Algunas experiencias pioneras en la región se ubican en Brasil en los ochenta, y en Uruguay y en Chile en los noventa. Pero, las experiencias más estudiadas son las que se establecieron en Chile y en Uruguay.

Un estado del conocimiento elaborado por Vercellino (2012) sobre esta cuestión apunta que una revisión de la bibliografía especializada sobre la extensión de las jornadas de trabajo apunta a tres amplias líneas de análisis: a) aquellos estudios que vinculan el tiempo que pasan los alumnos en la escuela y las distintas variables relacionadas con el resultado del aprendizaje; b) otro grupo de estudios busca dar cuenta de la puesta en marcha de esta política y los avances y avatares que enfrenta; y, c) finalmente, un conjunto de investigaciones analizan, desde una perspectiva crítica, la organización del tiempo escolar en estas experiencias educativas.

La primera línea de investigación apunta que los estudios se centran en tres orientaciones:

a) Uno, la cantidad de tiempo realmente asignado al aprendizaje en los salones de clase y la posibilidad de aprender de los estudiantes a lo largo de las jornadas ampliadas.

b) Dos, aquellos estudios que analizan el tiempo dedicado a una tarea escolar centrada en una actividad promovida por el docente que incentive el aprendizaje dentro del aula.

c) Tres, las investigaciones sobre el tiempo de aprendizaje académico; es decir, el tiempo empleado por el maestro en un aprendizaje de carácter académico (Vercellino, 2012).

Por otro lado, hay dos tendencias en los estudios del segundo grupo de investigaciones: por un lado, se identifican las diversas modalidades a través de las cuales 
La extensión de la jornada escolar en México y sus efectos en los maestros de primaria

se ha puesto en marcha este proyecto; $y$, por otro, se estudian los niveles de satisfacción de los destinatarios de estos programas ${ }^{2}$.

El tercer grupo de investigaciones apunta que se ha descuidado el análisis de la organización del tiempo clásico que, con esta reforma, busca modificar uno de los componentes estructurales más importantes de la vida escolar que se vinculan con los espacios escolares y los agrupamientos de los alumnos en las aulas de clase, entre otros aspectos.

Las ETC en México es un proyecto que se inició a finales de la década pasada y que cobró cuerpo con la Alianza por la Calidad de la Educación (ACE) 3 que firmó la dirigencia nacional del Sindicato Nacional de Trabajadores de la Educación (SNTE) con el gobierno federal. Este acuerdo recoge compromisos y obligaciones que México estableció con la Organización para la Cooperación y el Desarrollo Económicos (OCDE).

En diciembre de 2008 se emitieron las reglas de operación de las ETC. El objetivo declarado es generar condiciones institucionales que favorezcan la mejoría de los aprendizajes de los estudiantes de preescolar y primaria que contribuya a:

a) Asegurar el aprendizaje de los contenidos de los planes y programas de estudio vigentes.

b) Emplear tecnologías de la información y la comunicación para aprender a comprender.

c) Introducir las bases fundamentales para el aprendizaje del idioma inglés.

d) Desarrollar competencias para una vida saludable.

e) Potenciar la capacidad de comprensión del mundo natural y social (SEP, 2010).

La jornada laboral de los maestros es uno de los elementos más importantes que moldean con mucha fuerza la vida escolar. Este elemento junto a otros es el que da coherencia a las actividades escolares; entre las cuales destacan: la transmisión de saberes, la asignación de calificaciones, la distribución de los alumnos en las aulas, entre las más importantes.

El programa ETC, señala además un objetivo que permite observar otra lógica que subyace en él: un objetivo específico es: Ofrecer una opción para que los niños de las

${ }^{2}$ En relación con esta segunda línea de investigación un estudio (Department of Education, USA) citado en Vercellino (2012) sugiere tres áreas de investigación sobre este fenómeno: uno, la cantidad de trabajo llevado a cabo en la escuela en términos del impacto de éste con los resultados educativos; dos, la calidad del trabajo en las escuelas y fuera de ellas para complementar el aprendizaje; y, tres, la naturaleza de la concepción del tiempo que tienen los maestros en un contexto profesional de agrupación de alumnos diferentes y de distintas edades en un solo espacio. ${ }^{3} \mathrm{La}$ ACE es un documento en el cual se identifican las directrices más relevantes que la OCDE ha diseñado para América Latina y que se pueden resumir, en el caso de México, en: a) el establecimiento de nuevos criterios de selección de docentes; b) instauración de periodos de prueba obligatorios para los maestros; c) la certificación de los profesores; y, d) tomar medidas ante los maestros ineficaces (OCDE, 2004; 2010). 
familias que lo requieran, amplíen su estancia en el centro educativo a fin de fortalecer su formación integral y los padres de familia puedan desempeñarse laboralmente con mayor tranquilidad, elevar su capacidad productiva y fortalecer su realización personal (DOF, 25/02/2010: 2).

Así, este proyecto no solo tiene objetivos de tipo pedagógico sino también de carácter externo asociado a las condiciones laborales de los padres de familia que dedican tiempo al trabajo. Esto explica, como se verá en el análisis, que muchos padres apoyan este proyecto porque les permite mantener a sus hijos en las escuelas mientras ellos trabajan. Actualmente, las actividades según el programa se concluyen hasta las 17 horas. Véase cuadro uno:

\begin{tabular}{|l|l|}
\hline Horas & Actividades \\
\hline 13:00-14:00 horas & Alimentación \\
\hline 14:00-14:15 horas & Recreo \\
\hline 14:15-14:45 horas & Apoyo a tareas \\
\hline 14:45-15:15 horas & Recreación y convivencia \\
\hline 15:15-16:00 horas & TIC`s, educación artística e inglés \\
\hline 16:00-17:00 horas & Planeación y organización docente \\
\hline
\end{tabular}

Cuadro 1. Distribución de las actividades en las ETC (Fuente: SEP, 2010)

\section{Actividades pedagógicas}

La retórica oficial apunta que uno de sus propósitos centrales de las ETC es mejorar los aprendizajes de los alumnos mediante el alargamiento de las jornadas de trabajo ${ }^{4}$. En este sentido, es evidente que el supuesto que subyace en esta afirmación es que el docente al emplear más horas de trabajo contribuye significativamente en este importante objetivo.

Sin embargo, las prácticas pedagógicas de los maestros en las escuelas están estructuradas en razón de las prioridades que la SEP en México sobre las actividades de lectura, escritura y matemáticas. En esta perspectiva, esta política rompe la estructura tradicional de la jornada escolar y el resultado es que los maestros, o bien dirigen sus actividades hacia los niños que necesitan apoyo para mejorar en lectura, escritura y matemáticas o bien se orientan a otro tipo de actividades de orden cultural o deportivo.

Con respecto a la visión que tienen los maestros sobre la importancia pedagógica de las ETC el caso A señala: "[En principio] se quedan los niños que van mal [...] los atrasados $[. .$.$] pero también se quedan los que van bien [\ldots]$ estos niños no necesitan quedarse; pero se quedan".

\footnotetext{
4 El objetivo general del programa de ETC en México es: "Contribuir a fortalecer las oportunidades de mejorar el logro de aprendizaje de los estudiantes a través de la ampliación y uso eficiente del horario escolar en escuelas de educación básica" (DOF, 25/05/10:3).
}

Acta Hispanica, Hungría, Supplementum II: 835-845, 2020, ISSN: 1416-7263 | 839 
La extensión de la jornada escolar en México y sus efectos en los maestros de primaria

Las actividades que realizan los maestros con los niños después de las trece horas (que era la hora tradicional de la salida de los alumnos), también son sobre los mismos contenidos; sin embargo, se añaden clases de inglés y/o computación. El caso A apunta: "Se dan clases de inglés y computación; si es que hay computadoras; pero estas clases no son todos los días [solo tres días a la semana]; el resto de la jornada es para trabajar los contenidos de los programas de la SEP y sobre todo de los libros de texto"5.

El problema [de la ETC] señala el maestro del caso A: “[...] es que después de la una ya no sabemos qué hacer con ellos; se ponen muy inquietos... hacemos muchas actividades para llenar el horario". El caso B señala: "Por más que hacemos actividades los niños se aburren [...] y ya se quieren ir ¿Yo que hago? [...] Los saco del salón a pasear por la escuela, a veces corremos y después descansamos [...]"'.

Se identifica en estos testimonios una improvisación de las jornadas ampliadas cuando se observa que pueden, en algunos casos, impartirse clases de inglés o computación y en otros casos, no. Al respecto el caso B apunta: "Cuando el maestro de inglés o computación no llega, nosotros tenemos que continuar con las clases [...] Esto ocurre con frecuencia porque a los maestros a veces no les pagan los padres de familia o la propia SEP los cambia [...] esto nos provoca problemas".

El caso D, es una maestra ya mayor y comenta: "Nos obligaron a aceptar la ETC y [...] [además] nos dijeron que teníamos que hacer el diplomado7; porque en ese diplomado nos iban a decir que hacer en la jornada ampliada [...] Y resulta que fuimos y nada. Nos dijeron que teníamos que hacer actividades para cubrir la jornada".

Esta misma maestra agrega: "La verdad es que si [...] después de la una [las trece horas] los niños se ponen muy inquietos y ya se quieren ir. Yo les he dicho a las mamás que si quieren venir por sus niños que vengan y yo los dejo salir [...] Así me quedo con pocos y ya es más fácil el trabajo”.

\section{Desgaste y cansancio}

Importa aquí señalar que estas transformaciones de la vida escolar que impulsan los gobiernos latinoamericanos responden a un contexto caracterizado por una recesión económica. ${ }^{8}$

\footnotetext{
${ }^{5}$ La propuesta de la SEP en relación con las actividades de las ETC es, según su normativa: a) apoyo a tareas; b) recreación y convivencia; c) tecnologías de información y comunicación, inglés y educación artística; y d) planeación y organización de actividades docentes (SEP, 2010: 2).

${ }^{6}$ El caso D señala: "Este año me tocaron los niños de primer año; los más chiquitos [...] Antes de la una ya se quieren ir [...] Algunos hasta se duermen[...] Yo, a veces los llevo cargando a la dirección y los acuesto en el sillón hasta que termina la jornada".

${ }^{7}$ A raíz de la reforma educativa de primaria en 2009 se inició la capacitación a los maestros con un diplomado en Competencias a nivel de aula; que es el enfoque actual de la reforma.

${ }^{8}$ En efecto, las transformaciones más profundas al sistema educativo iniciaron con la crisis de la deuda externa de principios de los ochenta. En los noventa, con la firma del Acuerdo Nacional
} 
Gimeno (2009) apunta que hay ausencia de estudios sobre los impactos que la instauración de las ETC ha tenido en la fatiga de los alumnos o la relación entre tiempo escolar y tiempo extraescolar. A lo que hay que agregar el cansancio y la fatiga de los maestros. A este respecto, el caso B apunta: "En este año a mí me tocaron los más pequeños y los niños tardan mucho en acostumbrarse a estar en la escuela tanto tiempo".

El fenómeno es complejo porque involucra tanto a los niños y sus condiciones sociales y culturales como a los profesores de educación básica (preescolar y primaria) que son quienes los atienden en las escuelas públicas. Y en particular, los efectos que este proyecto tiene para los maestros. El caso A señala: [Después de la jornada] "los niños ya están cansados y se ponen muy inquietos; y nosotros... pues también ya estamos cansados [...] La jornada nos agota”.

El desgaste al que se refiere el caso B también lo señala el caso C: "[...] el cansancio es mucho; tanto para los niños como para [nosotros] los profesores [...] fíjese que al principio los niños lloraban mucho al medio día porque se querían ir a sus casas con sus papás [...]" (Caso C).

Uno de los mecanismos más importantes de la intensificación del trabajo se traduce en el alargamiento de las jornadas de trabajo que reducen el tiempo dedicado a la relajación y al descanso de los maestros provocando sobrecarga crónica en el trabajo reduciendo, contradictoriamente, la calidad del servicio y separando la ejecución del trabajo en sentido estricto de su concepción (Hargreaves, 2009).

El caso $C$ señala: "Mire [...] salgo muy temprano para llegar a la escuela[...] y salgo después de las tres y media[...] tomo el camión; porque no tengo carro y voy llegando a mi casa casi a las seis de la tarde; ya no puedo hacer nada y [además] llego muy cansado". Este mismo caso también señala que lo que más le afecta es el estrés?. Él apunta: “[...] es mucho tiempo el que estás en la escuela... Hay un descanso a las once de la mañana y otros diez minutos antes de la salida $[\ldots]$ ". 10 .

para la Modernización de la Educación Básica (ANMEB) se construyen las bases de estos cambios: a) la descentralización que responsabiliza a los estados de los sistemas educativos estatales; b) se impone un modelo de contención salarial con un programa de competitividad denominado: Carrera Magisterial (CM) para consolidar a mediano plazo los topes salariales. En 2008, con la firma de la ACE se imponen los estímulos a la calidad docente a través de exámenes estandarizados; y se establecen las ETC.

${ }^{9}$ Los factores que han provocado este tipo de padecimientos como el estrés y la angustia, entre otros, tienen como origen cuatro procesos derivados del modelo económico de desarrollo capitalista de las últimas décadas: uno, el incremento de la incertidumbre laboral de los trabajadores; dos, la creciente reducción o la pérdida total o parcial de las prestaciones; tres, el cambio de la lógica de la remuneración salarial basada en mecanismos de competitividad y eficiencia; y, cuatro, el subempleo, que, producto del crecimiento del desempleo (Cfr. De Vries y Wilkerson, 2003).

${ }^{10}$ Un dato importante sobre la relación entre la organización laboral y la salud mental de los trabajadores es que el alargamiento de las jornadas laborales influye negativamente en la salud física y mental de los trabajadores. Sin embargo, cuando la flexibilidad del horario es impuesta por 
La extensión de la jornada escolar en México y sus efectos en los maestros de primaria

Importa también señalar que, desde principios del siglo XXI la Organización Mundial de la Salud (OMS) ha señalado el incremento sistemático de las diversas enfermedades y trastornos mentales en la Población Económicamente Activa (PEA). Asimismo, también ha identificado que son los factores macroestructurales los que han elevado el riesgo de los trabajadores en general a padecer trastornos psíquicos asociados a la transición de las sociedades industriales a las sociedades de servicio.

El caso D también coincide con el caso C; la maestra señala: "Yo no vivo tan lejos de la escuela [donde trabajo] pero de todos modos llego después de la cuatro[...] y ya no me da tiempo de hacer otra cosa en mi casa. Estoy pensando en cambiarme de escuela [...] a una de doble turno ${ }^{11}$ para que no me quede hasta las tres y media".

\section{Percepciones salariales}

Las reformas de las dos últimas décadas del siglo XX provienen de la apertura comercial producto del proceso de globalización (Oman, 1994) cuya finalidad es atraer al capital extranjero. En estas transformaciones el Estado ha jugado un papel muy importante en términos de su retiro como agente regulador del crecimiento económico y de la distribución del ingreso. Este fenómeno ha significado que las antiguas desigualdades no solamente persistan; sino que se profundicen; y, además, se incrementen dentro de los mismos asalariados (Mora, 2010) ${ }^{12}$.

En el caso de los maestros el fenómeno se tradujo en la creación de un programa de competitividad y eficiencia denominado Carrera Magisterial (CM) con el cual se detuvieron los incrementos generalizados a los salarios. CM estableció incrementos solo para aquellos maestros que, mediante la evaluación a su trabajo, accedieran a aumentos de aproximadamente el 25 por ciento en cada una de las cinco categorías del programa. ${ }^{13}$

El caso D al respecto señala: "Nos dan un poco más de tres mil quinientos pesos [Sin embargo] nos descuentan el impuesto y pues ya nos queda menos. Es por decir: clase dada, clase pagada". El caso C apunta: "[...] no nos pagan las vacaciones ni los días que

la empresa el estrés laboral se incrementa en tanto que cuando el alargamiento de las jornadas laborales depende del trabajador no se observan efectos negativos (Linton, 2004).

${ }^{11}$ Las escuelas de doble turno son aquellas que funcionan en las mismas instalaciones. En el turno matutino, de 8 a 13 horas, y el vespertino, de 13 a 18 horas; lo cual impide que los maestros puedan quedarse en jornada ampliada.

${ }^{12}$ Los efectos de esta política de apertura comercial, traducida en políticas neoliberales, han sido, entre otros, la creciente presión sobre las condiciones laborales de los trabajadores; la cual ha cobrado cuerpo con dos mecanismos: uno, el control de las percepciones a través de topes salariales; $y$, dos, la disminución paulatina y sistemática de la protección social que ha profundizado la precarización (Castel, 2006; Carnoy, 2001).

${ }^{13}$ La política de contención salarial se refleja claramente en los bonos a los maestros en las ETC. Estos pagos no generan relación laboral con la SEP (DOF, 2010:5); y están sujetos al Impuesto Sobre la Renta (ISR). 
suspendemos [clases]. Agréguele que el pago nos lo retrasan [...] [Por ejemplo] ya estamos a fines de noviembre y apenas nos dan lo de septiembre y octubre".

Con respecto al pago el caso A señala: "Mire: al principio del año [escolar] tuvimos un desfile y ya no regresamos a la escuela [...] Y [Por este hecho] nos descontaron ochocientos pesos y nos pagan tres mil quinientos [...] haga usted la cuenta [...] " (Caso A).

En relación con los descuentos por inasistencias a la jornada completa el caso D apunta:

El fin de año íbamos a preparar nuestro convivio y le pedimos al director que nos autorizara salir pronto para el convivio. Y él nos dijo que no; que el convivio sería después de las tres y media. Nos opusimos y le dijimos que el convivio sería después de la una y que si quería nos mandara a descuento [...] Al final hicimos el convivio y no nos descontaron nada.

\section{Consideraciones finales}

Este estudio identifica algunos de los más importantes efectos que el proyecto de ETC ha tenido para los maestros de primaria en Tlaxcala. Por otro lado, ciertas consecuencias ya han sido identificadas por otras investigaciones, sin embargo, otras no. De manera que es necesario profundizar en cada uno de los aspectos que este trabajo muestra.

Una de las principales evidencias es que los estudios sobre este tema tocan distintos aspectos del fenómeno; sin embargo, lo que se observa es una ausencia de estudios que aborden aquellos niveles relacionados con los efectos que las ETC tienen para los profesores. En este sentido es que este análisis busca contribuir a avanzar en este aspecto.

Un asunto importante que este estudio destaca es que las transformaciones en la organización de la estructura escolar responden al proceso de cambio del modelo de acumulación de capital, vigente hasta los ochenta, que se ha traducido en la aplicación de políticas neoliberales que, en el caso estudiado, se reflejan en la extensión de las jornadas laborales.

Estos cambios en la reorganización de la estructura tradicional de las escuelas provocan conflictos en el desarrollo de las actividades que realizan los maestros. Se identifican también problemas derivados de la imposición de esta política porque se observa que el proyecto de ETC se estableció sin considerar consensos de los profesores; y además, problemas de carácter no solo administrativo sino pedagógico, entre otros. Los maestros enfrentan este proyecto con diferentes estrategias que nos indican que los docentes no responden a las exigencias que el proyecto impone.

Los aspectos pedagógicos están asociados a la ausencia de capacitación docente para trabajar en las ETC. Aquí es necesario profundizar en las variables que explican el fenómeno. En este mismo sentido, el desgaste y el cansancio que los maestros enfrentan en las ETC no han sido estudiados. En el caso del modelo de contención salarial ya tiene más de dos décadas de haberse impuesto a través de topes salariales; y con este esquema se profundiza la pérdida de prestaciones a los docentes. 
La extensión de la jornada escolar en México y sus efectos en los maestros de primaria

\section{Referencias bibliográficas}

Agamben, Giorgio (2011). ¿Qué es un dispositivo? Sociológica, 73. Asequible en: http://www.sociologicamexico.azc.uam.mx/index.php/Sociologica/article/view/112/ 103, fecha de consulta: 19-11-2019.

Baquero, Ricardo - Terigi, Flavia (1996). En búsqueda de una unidad de análisis del aprendizaje escolar. Apuntes pedagógicos. Buenos Aires: UTE/CTERA.

Bosco, Alejandra (2000). Los recursos informáticos en la tecnología organizativa y simbólica de la escuela. Estudio de un caso. (Tesis doctoral). Barcelona: Universidad de Barcelona.

Carnoy, Martín (2001). El trabajo flexible en la era de la información. Madrid: Alianza.

Castel, Robert (2006). La metamorfosis de la cuestión social. Una crónica del salariado. Buenos Aires: Paidós.

Centro de Implementación de Políticas Públicas para la Equidad y el Crecimiento (CIPPEC) (2006). Estudio para la implementación de una política nacional de extensión de la jornada escolar. Buenos Aires.

De Vries, Marten - Wilkerson, Bill (2003). Stress, Work and Mental Health: a Global Perspective. Acta neuropsychiatrica, XV.

Diario Oficial de la Federación (2010a). Lineamientos para la operación de las escuelas de tiempo completo. México.

Diario Oficial de la Federación (2010b). Programa nacional de escuelas de tiempo completo. México.

Dussel, Inés (2006). De la primaria a la EGB: ¿Qué cambió en la enseñanza elemental en los últimos años? En: F. Terigi (comp.). Diez miradas sobre la escuela primaria. Buenos Aires: Siglo XXI.

Gimeno Sacristán, José (2009). El valor del tiempo en educación, Madrid: Morata.

Hargreaves, Andy (2009). Profesorado, cultura y posmodernidad. Cambian los tiempos, cambia el profesorado. Madrid: Morata.

Hargreaves, Andy - Goodson, Ivor (2008). Educational Change Over Time? The Sustainability and Nonsustainability of Three Decades of Change and Continuity, EAQ Educational Administration Quarterly, vol. XLII, núm. 1. Asequible en: http://citeseerx.ist.psu.edu/viewdoc/download?doi=10.1.1.1000.5090\&rep=rep1\&typ $\mathrm{e}=$ pdf, fecha de consulta: 19-11-2019.

Linton, Steven (2004). Does Work Stress Predict Insomnia? A Prospective Study. British Journal of Health Psychology, IX.

Martinic, Sergio - Villalta, Marco (2013). La gestión del tiempo en la sala de clases y los rendimientos escolares en escuelas con jornada completa en Chile. Perfiles educativos, 147. UNAM. 
Mora, Minor - de Oliveira, Orlandina (2010). Las desigualdades laborales en México: evolución, patrones y tendencias. En: Cortés, Fernando - Orlandina de Oliveira, Desigualdad social. México: COLMEX.

OCDE (2010). Acuerdo de cooperación. México-OCDE para mejorar la calidad de las escuelas mexicanas. OCDE.

OCDE (2004). La cuestión del profesorado: atraer, capacitar y conservar a profesores eficientes, OCDE. Asequible en https://www.oecd.org/education/school/34991371.pdf, fecha de consulta: 19-11-2019.

Oman, Charles (1994). Globalization and Regionalization: the Challenge for Developing Countries, Paris: OCDE.

Ramírez, Fernando (2014). Sobre la extensión de la jornada escolar en Uruguay: ocho supuestos de la política educativa en debate. Revista Latinoamericana de Estudios Educativos, XLIV/ 2. México, CEE.

Romero, Claudia (2006). Funciones del asesoramiento escolar en los procesos de cambio educativo en la sociedad del conocimiento: Un estudio de caso en escuelas secundarias de la ciudad de Buenos Aires. (Tesis doctoral). Madrid: Universidad Complutense de Madrid, Madrid. Asequible en: http://webs.ucm.es/BUCM/tesis/edu/ucm-t29346.pdf, fecha de consulta: 19-11-2019. SEP (2010). Lineamientos para la operación del programa de jornada ampliada en educación preescolar y primaria. México.

Tyack, David - Cuban, Larry (1995). En busca de la utopía. Un siglo de reformas de las escuelas públicas, México: Fondo de Cultura Económica.

Vercellino, Soledad (2012). La ampliación del tiempo escolar: ¿Se modifican los componentes duros del formato escolar? Revisión bibliográfica sobre estas temáticas. Revista electrónica Educare, XVI/3. Asequible en: https://www.revistas.una.ac.cr/index.php /EDUCARE/article/view/4773/16014, fecha de consulta: 19-11-219. 